\title{
Trends to Acid-Labile Cys Protecting Groups: Thp as an Efficient and Non-Aromatic Cys Protecting Group for Fmoc Chemistry
}

\author{
Iván Ramos-Tomillero ${ }^{1-3}$, Hortensia Rodríguez ${ }^{4}$, and Fernando Albericio ${ }^{1-5}$ \\ ${ }^{1}$ Institute for Research in Biomedicine; ${ }^{2}$ Department of Organic Chemistry, University of Barcelona; \\ ${ }^{3}$ CIBER-BBN, 08028, Barcelona, Spain; ${ }^{4}$ School of Chemistry, Yachay Tech, Urcuqui, Ecuador; \\ ${ }^{5}$ School of Chemistry University of KwaZulu Natal Durban, 4000, South Africa
}

\section{Introduction}

Since the discovery of solid-phase peptide synthesis (SPPS) by Merrifield [1], the peptide field has suffered a dramatic change in a wide set of points such as the development of new solid supports, the discovery of highly efficient coupling reagents and additives, as well as the fashionable design of linkers and protecting groups. Consequently, the solid phase peptides synthesis is considered the most efficient way to synthesize a broad number of complex molecules.

Into the complete process of the SPPS, a large number of problematic side reactions occur, in consequence during the past decades tremendous efforts have been done in order to avoid this drawbacks that jeopardize the efficiency of this methodology. Amino acid racemization is still an issue in SPPS because of the similarity between the desired product and the concomitant racemic product. In this sense, several factors such as the coupling reagents, the handles or the protecting groups, or even the basic conditions used during the peptide synthesis, could affect directly the integrity of the $\alpha$-proton, which are able to modulate the amino acid racemization.

\section{Cysteine}

In nature, cysteine (Cys) is crucial due to its capacity to confer stability to peptides and proteins, but also constrain the specific conformation of these biomolecules through disulfide bonds [2,3]. In addition, its use has modernized the chemical synthesis of large polypeptides and proteins by Native Chemical Ligation [4]. Accordingly, Cys is considered one of the most problematic amino acid in SPPS, due to the high instability of its $\alpha$-proton caused by the proximity of the thiol group, which promotes the racemization of its chiral center. Furthermore, $\beta$-elimination, oxidation and alkylation are other examples of potentials Cys side reactions. All these Cys collateral reactions are responsible of the complex peptide crude products obtained during the synthesis of Cys-rich peptides [5]. Nowadays, extensive possibilities to avoid these Cys promoted side reactions have been studied. In that sense, the development of new coupling reagents and additives for SPPS, assisted the by-products minimization [6]. Additionally, special efforts were focused into Cys side chain protection and as a result, a myriad of Cys protecting groups stable to a set of chemical conditions have been developed for their use in SPPS [7].

\section{Protection schemes}

The stability and lability of protecting groups play an important role into the strategy selection criteria for SPPS. While Boc chemistry requires base-labile protecting groups, the Fmoc/tBu strategy involves the use of acid cleavable groups. The conferred stability is related by the kind of binding between the $\beta$-thiol group of Cys and the protecting group. As reported in the literature, thioether linkage is the most commonly used thiol protection form, due to the stability under several conditions. Thus, in recent years the development of the most successful protecting groups for Cys are based on this special linkage. In this regard, herein we will focus our attention in acid-labile Cys protecting groups in general, and in the recently developed non-aromatic acid-labile Cys protecting group, in particular.

\section{Acid-labile Cys protecting groups}

The conscious design of protecting groups had triggered a myriad of groups, which are stable in a broad range of acidic conditions (Figure 1). Therefore, the selective protecting group elimination is possible, depending on the acidic conditions used. As a result of years of studies in the field, there are several Cys protecting groups available; some of them are labile in low acid concentration such as 
Mmt [8], Trt [9-11] or Thp [12,13]; others are more stable in low concentrations of trifluoroacetic acid (TFA) but cleavable using higher quantity of TFA - Diphenylmethyl (Dpm) $[9,11,14], t \mathrm{Bu}[15,16]$ or MBom [17] and moreover, there are groups which are completely stable to TFA and removable using harsh conditions such as HF, for example Bom [18], Meb or Mob [19-21] groups.

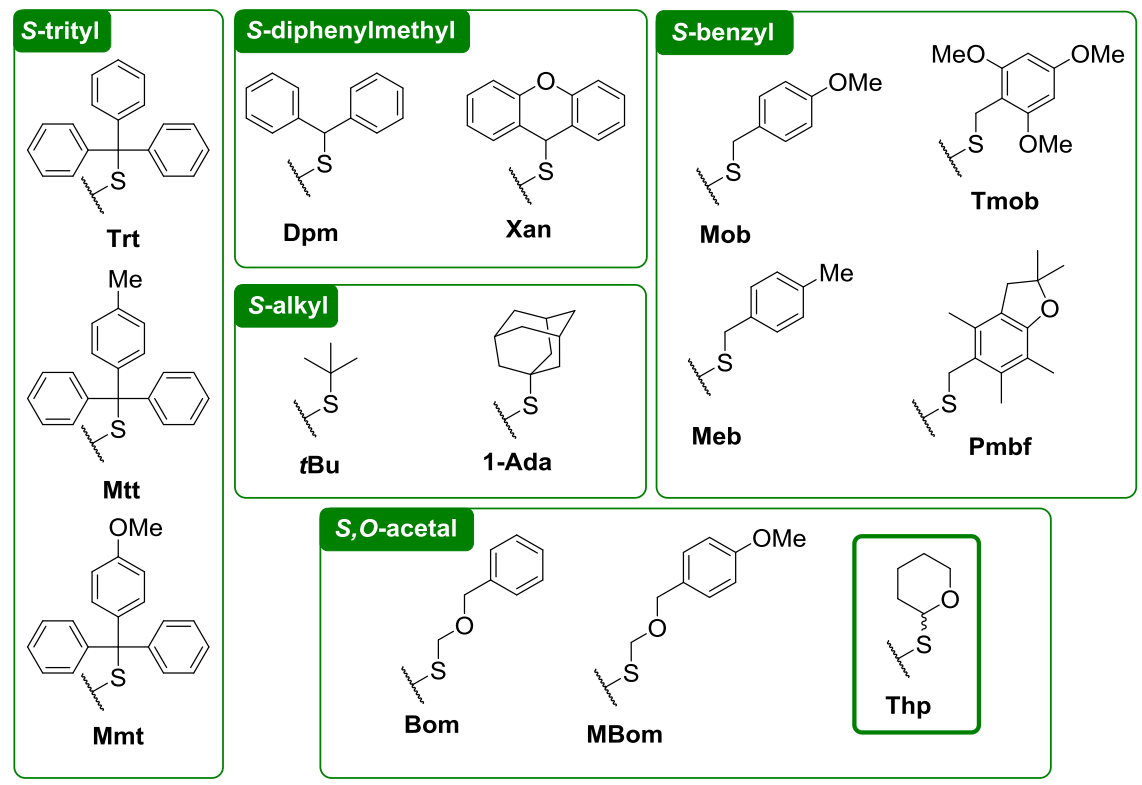

Fig. 1. Acid-labile Cys protecting groups.

Due to their chemical properties, most protecting groups and linkers used in SPPS [22] are based on benzyl, diphenylmethyl, and triphenylmethyl structures. The mechanisms of elimination are based on the protonation of the thiol group, and followed by the carbocation formation. Herein, the stability of the liberated carbocation in acidic conditions manages the protecting group lability. Consequently, the more acid labile the more stable carbocation [23]. Some factors contribute to their stability such as steric hindrances or field-inductive effects, but the most imperative effect correspond to the electronic delocalization, which increase the carbocation stability [24].

\section{New generation of Cys protecting groups}

Although trityl-, diphenylmethyl-, and benzyl-like scaffolds are the most used for the protection of Cys, in the recent years the studies with $\mathrm{S}, \mathrm{O}$-acetal protecting groups has presented extremely low racemization levels [25]. While benzyloxymethyl (Bom) is used for Boc chemistry, 4-methoxybenzyloxymethyl (MBom) and the recently published tetrahydropyranyl (Thp) are compatible with the Fmoc/tBu approach. Regarding the molecular structures (Figure 1), most of the protecting groups available contain aromatics rings, except $t \mathrm{Bu}, 1$-Ada and Thp. In that sense, in the production of protected hydrophobic peptides, the use of bulky protecting groups in SPPS, such as trityl-like scaffolds as well as $t \mathrm{Bu}, 1$-Ada, affects their inter/intrachain interaction during peptide elongation and this fact jeopardize the purity of the final product.

Apart of its recent application in Fmoc chemistry [26], Thp is extensively used as hydroxyl protecting group [27-30] as well as a linker [31-34], due to its chemical properties and its stability in strong bases, and acylating and alkylating agents among others. Accordingly, Thp is an excellent candidate for use in SPPS, but it is known that the main drawback should be the formation of a new stereocenter that leads to diastereomeric mixtures. Nevertheless, as with other protecting groups or linkers, their use is temporary. Consequently, the formation of the stereocenter is not a limitation. 


\section{Tetrahydropyranyl (Thp) for Fmoc peptide chemistry}

Seeking a protecting group which will be able to decrease the racemization to minimum levels, MBom and Thp could be the choice for Fmoc chemistry. While the MBom presents a straightforward preparation of the protected amino acid unit, Thp is easily introduced into the Cys thiol group by the using a catalytic amount of acid in presence on dihydropyran. Regarding the final protecting group elimination (Figure 2), in both cases an aldehyde is generated, formaldehyde and 5-hydroxypentanal for MBom and Thp, respectively.

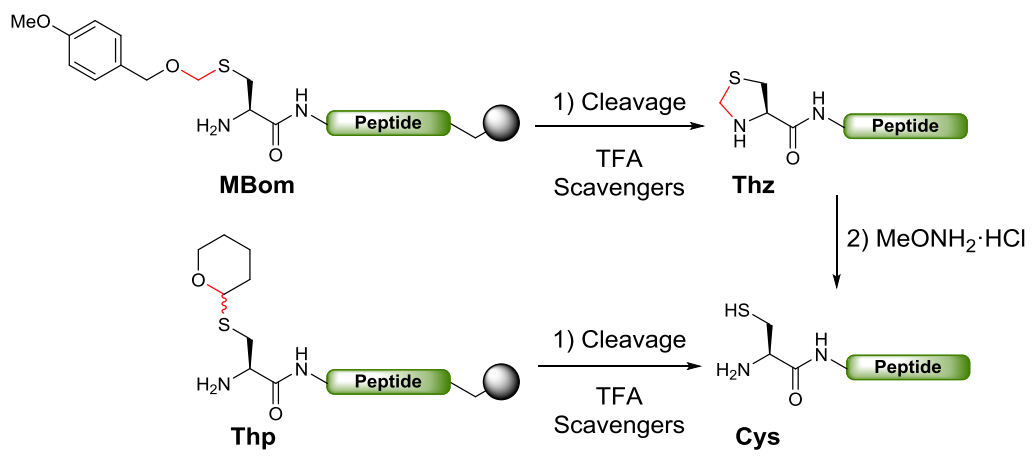

Fig. 2. N-terminal Cys protecting group elimination.

Accordingly, the formaldehyde formation supposes an important drawback because of the hydroxymethylation. An undesirable side reaction is the formation of the thiazolidine (Thz) ring in $N$-terminal Cys. In that sense, as it was demonstrated for Thp in their use for $N$-terminal peptides Cys containing peptides [26], the formation of 5-hydroxypentanal does not create a problem due to the fact that the hydroxyl group present in the molecule, suffer an intramolecular hydroxymethylation, trapping

Table 1. Cys racemization studies for AlaCys-Leu tripeptide.

\begin{tabular}{ccc}
\hline Resin & Cys PG & \% Racemization $^{a}$ \\
\hline \multirow{3}{*}{ Sieber Amide } & Thp & $0.7 \%$ \\
& Trt & $3.3 \%$ \\
& Dpm & $6.8 \%$ \\
Rink Amide & Thp & $0.5 \%$ \\
& Trt & $2.3 \%$ \\
2-Chlorotrityl & Thp & $0.2 \%$ \\
chloride & Trt & $0.7 \%$ \\
\hline
\end{tabular}

${ }^{a}$ Calculated as (D-peptide peak area /L-peptide peak area) $\times 100$ the reactive electrophilic carbon atom.

Furthermore, use of these protecting groups in a peptide sequence or $C$-terminal Cys containing peptides results in extremely low racemization levels $(<1 \%)$ for the synthesis of model tripeptides Ala-Cys-Leu (Table 1). Additionally, when Cys is directly attached to the resin forming an ester bond, Cys racemization and the $C$-terminal 3-(1-piperidinyl)-alanine formation [35] occurs in a high level during the SPPS process, destroying the integrity of the desired peptide. In that sense, the use of Thp as a Cys protecting group, decrease even those racemization of the Cys $\alpha$-proton and also the $\beta$-elimination followed by piperidine addition, obtaining the undesired 3-(1-piperidinyl)alanine adduct.

\section{Conclusions}

The methodological spectrum available for the synthesis of peptides in solid supports, has suffered during several years an impressive transformation. Always seeking to avoid the side reactions, which make the synthesis of this kind of special molecules difficult. In that sense, all the scientific contributions in the field make the SPPS an outstanding and rigorous way to obtain the myriad of interesting small molecules, peptides or proteins. Our recent contribution to the field, the Cys protecting group Thp, demonstrated that now with a lot of work done in the field, it is possible to 
obtain extremely low racemization levels $(<1 \%)$. Due to the scientific ambition, the field is tending to reach the synthetic perfection. The question is: will it be possible?

\section{Acknowledgments}

I. R-T. thanks the Generalitat de Catalunya for a predoctoral fellowship. This work was funded in part by the following: the CICYT (CTQ2012-30930), the Generalitat de Catalunya (2014 SGR 137), the Institute for Research in Biomedicine Barcelona (IRB Barcelona) (Spain).

\section{References}

1. Merrifield, R.B. J. Am. Chem. Soc. 85, 2149-2154 (1963), http://dx.doi.org/10.1021/ja00897a025

2. Góngora-Benítez, M., et al. Chem. Rev. 114, 901-926 (2013), http://dx.doi.org/10.1021/cr400031z

3. Postma, T.M., et al. Eur. J. Org. Chem. 2014, 3519-3530 (2014), http://dx.doi.org/10.1002/ejoc.201402149

4. Dawson, P.E., et al. Science 266, 5186, 776-779 (1994), http://dx.doi.org/10.1126/science.7973629

5. Stathopoulos, P., et al. Amino Acids 44, 1357-1363 (2013), http://dx.doi.org/10.1007/s00726-013-1471-7

6. Han, S.-Y., Kim, Y.-A. Tetrahedron 60, 2447-2467 (2004), http://dx.doi.org/10.1016/j.tet.2004.01.020

7. Isidro-Llobet, A., et al. Chem. Rev. 109, 2455-2504 (2009), http://dx.doi.org/10.1021/cr800323s

8. Barlos, K., et al. Int. J. Pept. Protein Res. 47, 148-153 (1996), http://dx.doi.org/10.1111/j.1399-

3011.1996.tb01338.x

9. Zervas, L., Photaki, I. J. Am. Chem. Soc. 84, 3887-3897 (1962), http://dx.doi.org/10.1021/ja00879a019

10. Hiskey, R.G., Adams, J.B.J. J. Org. Chem. 30, 1340 (1965), http://dx.doi.org/10.1021/jo01015a567

11. Photaki, I., et al. J. Chem. Soc. C Org. 19, 2683-2687 (1970), http://dx.doi.org/10.1039//339700002683

12. Holland, G.F., et al. J. Am. Chem. Soc. 80, 3765-3769 (1958), http://dx.doi.org/10.1021/ja01547a075

13. Ramos-Tomillero, I., et al. Org. Lett. 17, 1680-1683 (2015), http://dx.doi.org/10.1021/acs.orglett.5b00444

14. Góngora-Benítez, M., et al. Org. Lett. 14, 5472-475 (2012), http://dx.doi.org/10.1021/ol302550p

15. Chimiak, A. Rocz. Chem. 38, 883-885 (1964).

16. Pastuszak, J.J, et al. J. Org. Chem. 46, 1868-1873 (1981), http://dx.doi.org/10.1021/jo00322a024

17. Hibino, H., Nishiuchi, Y. Org. Lett. 14, 1926-1929 (2012), http://dx.doi.org/10.1021/ol300592w

18. Otaka, A., et al. Chem. Pharm. Bull. 37, 526-528 (1989), http://dx.doi.org/10.1248/cpb.37.526

19. Akabori, S., et al. Bull. Chem. Soc. Jpn. 37, 433-434 (1964), http://dx.doi.org/10.1246/bcsj.37.433

20. Erickson, B.W., et al. J. Am. Chem. Soc. 95, 3750-3756 (1973), http://dx.doi.org/10.1021/ja00792a 046

21. Live, D.H., et al. J. Org. Chem. 42, 3556-3561 (1977), http://dx.doi.org/10.1021/jo00442a025

22. Guillier, F., et al. Chem. Rev. 100, 2091-2157 (2000), http://dx.doi.org/10.1021/cr980040+

23. Ramos-Tomillero, I., et al. Molecules 18, 5155-5162 (2013), http://dx.doi.org/10.3390/molecules 18055155

24. Hoffmann, R., et al. J. Phys. Chem. 73, 1789-1800 (1969), http://dx.doi.org/10.1021/j100726a027

25. Hibino, H., et al. J. Pept. Sci. 20, 30-35 (2014), http://dx.doi.org/10.1002/psc.2585

26. Ramos-Tomillero, I., Rodríguez, H. \& Albericio, F. Tetrahydropyranyl (Thp), a Non-Aromatic Acid-Labile Cys Protecting Group for SPPS. Proceedings of $24^{\text {th }}$ American Peptide Symposium. 2015, Poster YI-404

27. Miyashita, M., et al. J. Org. Chem. 42, 3772-3774 (1977), http://dx.doi.org/10.1021/jo00443a038

28. Bernady, K.F., et al. J. Org. Chem. 44, 1438-1447 (1979), http://dx.doi.org/10.1021/jo01323a017

29. Liu, J., et al. Tetrahedron Lett. 43, 4037-4039 (2002), http://dx.doi.org/10.1016/S0040-4039(02)00740-2

30. Stephens, J.R., et al. Eur. J. Org. Chem. 2003, 3827-3831 (2003), http://dx.doi.org/10.1002/ejoc.200300295

31. Thompson, L.A., et al. Tetrahedron Lett. 35, 9333-9336 (1994), http://dx.doi.org/10.1016/S00404039(00)78535-2

32. Smith, A.L., et al. Tetrahedron Lett. 39, 8317-8320 (1998), http://dx.doi.org/10.1016/S00404039(98)01824-3

33. Basso, A., Ernst, B. Tetrahedron Lett. 42, 6687-6690 (2001), http://dx.doi.org/10.1016/S00404039(01)01368-5

34. Torres-García, C., et al. Int. J. Pept. Res. Ther. 18, 7-19 (2011), http://dx.doi.org/10.1007/s10989-011-9274-8

35. Lukszo, J., et al. Lett. Pept. Sci. 3, 157-166 (1996), http://dx.doi.org/10.1007/BF00132978 